\title{
Calibration and Removal of Lateral Chromatic Aberration in Images
}

\author{
John Mallon * Paul F. Whelan \\ Vision Systems Group, Dublin City University, Dublin 9, Ireland
}

\begin{abstract}
This paper addresses the problem of compensating for lateral chromatic aberration in digital images through colour plane realignment. Two main contributions are made: the derivation of a model for lateral chromatic aberration in images, and the subsequent calibration of this model from a single view of a chess pattern. These advances lead to a practical and accurate alternative for the compensation of lateral chromatic aberrations. Experimental results validate the proposed models and calibration algorithm. The effects of colour channel correlations resulting from the camera colour filter array interpolation is examined and found to have a negligible magnitude relative to the chromatic aberration. Results with real data show how the removal of lateral chromatic aberration significantly improves the colour quality of the image.
\end{abstract}

Key words: Lateral chromatic aberration, Image warping, Camera calibration

\section{Introduction}

An optical instrument is required to faithfully produce a geometrically consistent image of a given object, where each point of the latter is imaged as a point in the image. The departure of practical optical systems from this ideal (gaussian or first order) behaviour is due to aberrations. In general it is impossible to design a system which is free from all aberrations. This leads lens manufacturers to consider aberration compensation as an optimisation between different types. We are interested in chromatic aberrations that have recently become more amplified due to the higher resolution sensors currently employed in many consumer and scientific cameras. By compensating for these

* Corresponding author. Tel.: +353 17005869 Fax: +353 17005508

Email address: john.mallon@eeng.dcu.ie (John Mallon). 
aberrations as a post process in the image array, higher quality images can be produced without recourse to expensive optics.

In a colour camera's lens, polychromatic light is split into a set of rays or wavelengths. Whilst traversing the optical system light of different wavelengths will follow slightly different paths. Upon reaching the image plane their misaligned recombination introduces chromatic aberration. Chromatic Aberration (CA) can be broadly classified as Axial Chromatic Aberration (ACA) (also known as Longitudinal CA) and Lateral Chromatic Aberration (LCA) (also known as Transverse CA). ACA arises from the longitudinal variation of focal position with wavelength along the optical axis. LCA is the variation of image size with wavelength or the vertical off-axis distance of a point from its prescribed point. In an image it is identified by a radially dependent misalignment of the colour planes. Chromatic aberrations are moving out of the sub-pixel range with the advent of high resolution arrays, giving rise to noticeable colour fringes at edges and high contrast areas. This gives the overall impression of poor quality or definition. Many consumer cameras display this aberration. For scientific applications, it is akin to the effects of colour shifts and blurring, that contravene the imaging models. We consider the digital compensation of LCA through image warping. There are two main aspects of digital compensation in images: determining what quantity of warp to apply, and the actual implementation of the warp. Our main contribution deals with the former problem, which has currently not been addressed, by considering the modelling and model calibration of LCA in images.

Chromatic aberration has been predominately studied with respect to image formation in the areas of microscopy, photogrammetry and computer vision, though recent advances in digital imaging has seen the emergence of commercial interest. Willson (1994) and Willson and Shafer (1991) considers an active lens control system to compensate for both LCA and ACA, by separately adjusting three RGB filter lenses to match the colour planes. Their work shows that chromatic aberrations can be compensated in an image by re-alignments of the colour channels. Boult (1992) formulates the compensation of LCA as an image warping problem. No aberration models are employed, focusing solely on the warping problem, and correcting based only on interpolation between control points. Jackowski et al. (1997) presents a similar study on geometric and colour correction in images based on a comparison with a well defined colour calibration chart. The models used are again surface approximations, which are far from optimal solutions, especially since only a limited number of control points are available to estimate the surface parameters. An algorithm for the measurement and compensation of both LCA and ACA has been proposed for fluorescence microscopy by Kuzubek and Matula (2000), and for electron microscopes by Freitag et al. (2005). These techniques are not transferrable to images acquired with regular imaging systems. With the goal of developing an auto-focus or depth indicator, Garcia et al. (2000) measure the 
blurring resulting from ACA on different colour planes around step edges.

Our proposed compensation is achieved by realigning the colour planes through image warping. Firstly, an LCA model is derived to precisely model the aberration over the entire image surface. This offers a more precise and concise means of extending the aberration, measured over a limited set of control points, to every pixel in the colour plane. LCA is initially measured by extracting the intersections of a chessboard pattern on each colour plane. No special planarity constraints or canonical representation of the pattern is required. It can be imaged without knowing its 3D position. Additionally, many of the target images are likely to be acquired with conventional digital cameras. These images are typically captured using a colour filter array, who's measurements are then interpolated to form the full colour image. Thus, we investigate if there is significant correlation between individual colour channels resulting from the interpolation process that may lead to additional chromatic distortion in regions of high chromatic content.

The measurement errors are filtered by non-linear least square fitting of the proposed LCA model. The partial derivatives of the quadratic cost functions are given allowing the exact computation of the gradients and Hessian matrices used by the optimisation algorithms. This gives a computational advantage over numerical estimation techniques. The suitability and stability of the model is examined by computing the uncertainties associated with the estimated parameters. The detailed results clearly demonstrate the successful compensation of LCA for real scenes.

\section{Geometrical Theory of Aberrations}

Optically, aberrations are compensated for by adding lens elements with appropriate properties. Chromatic aberration is typically eliminated for two selected wavelengths, but only at the center and some zonal region. These lenses are known as achromatised. Lenses corrected for three different wavelengths are known as apochromatic while superachromatic lenses are corrected for four wavelengths. We are interested in the remaining chromatic aberrations, known as the secondary spectrum. No distinctions are made between types of corrected lenses, as the derived models are generally applicable. Lateral chromatic aberration can be considered as the sum of two aberrations: lateral colour distortion due to the refraction index of the lens elements and the chromatic variation of distortion (Kingslake, 1978). We proceed by determining an appropriate model for the chromatic variation of distortion in the image plane. 


\subsection{Chromatic variation of distortion}

On the image plane, ideal image points are denoted in Euclidian space as $\mathbf{p}=[x, y]^{T}$ while actual points are $\breve{\mathbf{p}}=[\breve{x}, \breve{y}]^{T}$. Within the accuracy of gaussian optics or perfect projection $\mathbf{p}=\breve{\mathbf{p}}$. Referring to figure $1, \xi$ and $\eta$ are $\mathbf{x}$, $\mathrm{y}$ measurements in the plane of the aperture. As a consequence of the preceding optics, a wave other than the prescribed gaussian one is formed. This wave aberration can be expressed in polynomial form, derived from Seidel perturbation eikonals (Born and Wolf, 1980; Driscoll, 1978). In a general sys-

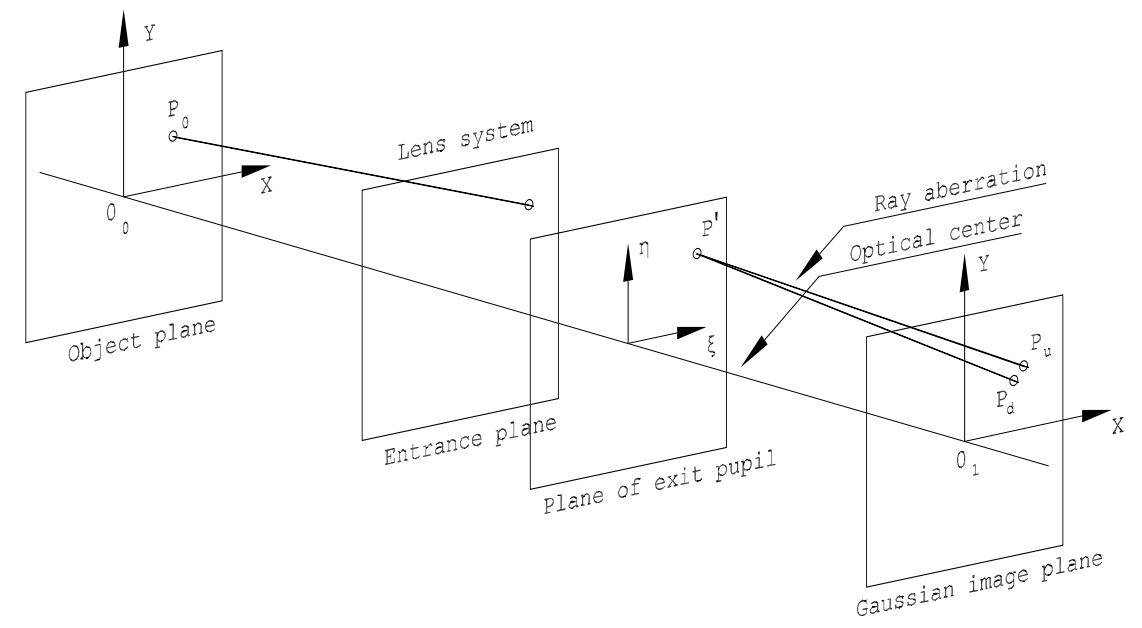

Fig. 1. General optical setup.

tem containing a number of refracting surfaces, the primary aberrations equal the sum of the corresponding aberration coefficients associated with the individual surfaces of the system. By this reasoning, aberrations are optically compensated for by the addition of appropriate lens elements. If decentering or misalignments of the surfaces is considered, subsequent image deformation may be approximated by perturbing the intermediately formed image by $x_{1} \rightarrow x_{1}+\lambda$ and $y_{1} \rightarrow y_{1}+\mu$. Considering the distortion component of the wave aberration equation, the corresponding wave aberration for the combined surfaces to a fourth order approximation is:

$$
\phi=k_{1} r^{2} \kappa^{2}+\lambda k_{1}\left(\xi\left(3 x^{2}+y^{2}\right)+2 \eta x y\right)+\mu k_{1}\left(\eta\left(3 y_{u}^{2}+x_{u}^{2}\right)+2 \xi x_{u} y_{u}\right),
$$

where $r^{2}=x^{2}+y^{2}$ and $\kappa^{2}=x \xi+y \eta$. The constant $k_{1}=E_{1}+\ldots E_{n}$ is the sum of the individual lens contributions. The combined decentering effects of multiple lens elements also sums in a linear fashion. The altered wavefront is the root of all aberrations formed on the image by distorting the ray projections. These ray aberrations are evaluated as the shift from the predicted gaussian coordinates by $\Delta_{x}=x-\breve{x}=\frac{\partial \phi}{\partial \xi}$ and $\Delta_{y}=y-\breve{y}=\frac{\partial \phi}{\partial \eta}$ (Born and Wolf, 1980). Evaluating this using the fourth order approximation of $\phi$, results in the combined cartesian model of distortion. 


$$
\begin{aligned}
& \mathcal{C}_{\mathcal{D}}(\mathbf{p}, \mathbf{k})_{x}=k_{1} x r^{2}+p_{1}\left(3 x^{2}+y^{2}\right)+2 p_{2} x y \\
& \mathcal{C}_{\mathcal{D}}(\mathbf{p}, \mathbf{k})_{y}=k_{1} y r^{2}+2 p_{1} x y+p_{2}\left(3 y^{2}+x^{2}\right),
\end{aligned}
$$

where $\mathbf{k}=\left[k_{1}, p_{1}, p_{2}\right]^{T}$ are the parameters, $p_{1}=\lambda k_{1}$ and $p_{2}=\mu k_{1}$. In this function the radial component is represented by $k_{1}$, while the distortions introduced by decentering correspond to $p_{1}$ and $p_{2}$. This decentering model is equivalent to that of Conrady (1919) as promoted by Brown (1966), found through exact ray tracing techniques. In many lenses a secondary chromatic spectrum exhibits a decentering form. Its inclusion leads to a more general model and improved modelling accuracy. This is investigated further in section 4 .

\subsection{Lateral colour distortion}

In addition to the chromatic variation of distortion there is an additional lateral colour distortion that is due to the refraction index variation of the lens elements. The refraction index is quite linear within the visible spectrum Kingslake (1978), resulting in the addition of an extra first order term that does not appear in the chromatic distortion equation. Deviations from linear behaviour are naturally accounted for in the chromatic distortion equation. Thus, the combined LCA for a specific frequency $(g)$, can thus be modelled as a function of another frequency $(f)$ by the addition of the chromatic variation

of distortion and the lateral colour distortion as:

$$
\begin{aligned}
& \mathcal{C}^{g}\left(\mathbf{p}_{f}, \mathbf{c}_{g}\right)_{x}=c_{1} x_{f}+c_{2} x_{f} r_{f}^{2}+c_{3}\left(3 x_{f}^{2}+y_{f}^{2}\right)+2 c_{4} x_{f} y_{f} \\
& \mathcal{C}^{g}\left(\mathbf{p}_{f}, \mathbf{c}_{g}\right)_{y}=c_{1} y_{f}+c_{2} y_{f} r_{f}^{2}+2 c_{3} x_{f} y_{f}+c_{4}\left(3 y_{f}^{2}+x_{f}^{2}\right),
\end{aligned}
$$

where $\mathbf{c}_{f}=\left(c_{1}, c_{2}, c_{3}, c_{4}\right)^{T}$ is the parameter vector.

\section{Model Calibration}

Lateral chromatic aberration is modelled for a specific frequency according to equation 2 . The actual secondary spectrum is difficult to exactly quantify, but manifests itself by misalignments in the colour planes as demonstrated by Willson (1994). The colour cameras used in these earlier works typically consisted of beam splitters with three RGB CCD elements. With the current standard digital technology, an image is formed with one CCD using a Colour Filter Array (CFA), such as the popular Bayar array. Thus, for each location in the image, two colours must be interpolated from the neighboring locations. This interpolation process is also referred to a demosaicking, see Popescu 
and Farid (2005); Ramanath et al. (2002) for an overview of methods. Due to this interpolation, it is quite possible that correlation may be introduced between separate colour planes, indeed many demosaicking methods explicitly use additional separate colours to improve their results. However, if the colours are not properly aligned (due to chromatic aberration) this can lead to more colour distortion being introduced, depending on the chromatic content of the light ray. The extent of this correlation is investigated in section 4 .

The RGB colour space is used in this work, due to its direct correspondence with wavelength. The chromatic aberration is compensated for by re-aligning two of the colour planes with the third reference plane. This reference plane is chosen as the green plane, which corresponds to the midpoint of the visible spectrum. It is also used in demosaicking methods as a reference or luminance channel.

\subsection{Measuring lateral chromatic aberrations}

Chromatic aberration has previously been measured by Kuzubek and Matula (2000) using florescent dyed beads. These are then imaged in 3D, when their centroids are estimated. From these centroids the LCA and ACA are measured. This approach is only suited to fluorescent microcopy, but the measured LCA exhibits a similar profile to the results obtained using our approach. Willson Willson (1994), measures chromatic aberration by comparing the location of edges detected on three colour planes. In this paper lateral chromatic aberration is measured by detecting the intersections of a chessboard pattern for each of the colour planes. These are automatically extracted by a two stage process of initial detection and sub-pixel refinement.

Initial estimates for the location of chessboard type intersection are obtained using standard corner detectors such as those described in Lucchese and Mitra (2002); Jain et al. (1995). For real situations where additional corners are detected, a further refinement step is necessary to remove false hits. A small $N \times N$ region of interest, $\Psi$, centered on the candidate corner is first thresholded using the mean gray level of $\Psi$. A symmetry measure $t_{\Psi}$ can then be calculated as:

$$
t_{\Psi}=\sum_{v=0}^{N} \sum_{u=0}^{N} \mathcal{O}(u, v), \text { where } \mathcal{O}(u, v)=\left\{\begin{array}{cc}
a & \begin{array}{c}
\Psi(u, v)=\Psi(N-u, N-v) \\
\Psi(u, v) \neq \Psi(u, N-v)
\end{array} \\
b & \text { otherwise }
\end{array}\right.
$$

where $(u, v)$ are the pixel coordinates, $a$ and $b$ are positive and negative constants. We obtain good performance using $a=6$ and $b=-1$ with $N=9$. High values of the symmetry measure $t_{\Psi}$ indicate the corner is situated on a chessboard intersection. 
For each colour plane the initial corner estimate is refined using a small $N \times N$ region of interest $\Psi$. A bilinear quadratic function is linearly fit to the intensity profile:

$$
\min _{\mathbf{s}}\left\|s_{1} u^{2}+s_{2} u v+s_{3} v^{2}+s_{4} u+s_{5} v+s_{6}-\Psi(u, v)\right\|^{2} .
$$

The intersection point or saddle point is derived from this surface as the intersection of the two lines $2 s_{1} u+s_{2} v+s_{4}=0$ and $s_{2} u+2 s_{3} v+s_{5}=0$. No special data ordering is necessary as comparisons are not made to external coordinate system. The accuracy of this detection method is examined in section 4 .

\subsection{Chromatic parameter estimation}

The pattern intersection points are represented in pixel coordinates as $\mathbf{m}_{f}=$ $\left(u_{f}, v_{f}\right)^{T}$ for a certain colour plane $f$. Given the image width and height as $w$ and $h$, the intersection coordinates are normalised by scaling $\overline{\mathbf{m}}_{f}=$ $\left(u_{f}, v_{f}\right)^{T} / s=\left(\bar{u}_{f}, \bar{v}_{f}\right)^{T}$, where $s=(w+h) / 2$. The normalised optical axis or center point of the apparent aberration, $\left(\dot{x}_{f}, \dot{y}_{f}\right)^{T}$, also needs to be estimated, as it generally does not lie at the center of the image. It is initially estimated as $\dot{x}_{f}=-w / 2 s$ and $\dot{y}_{f}=-h / 2 s$. For some cases an aspect difference $(a)$ also needs to be estimated. Fully scaled points on a certain colour plane are thus denoted by:

$$
\mathbf{p}_{f}=\left(x_{f}, y_{f}\right)^{T}=\left(a \bar{u}_{f}+\dot{x}_{f}, \bar{v}_{f}+\dot{y}_{f}\right)^{T},
$$

where $f$ denotes the colour plane, in this case either red $(r)$, blue $(b)$ or green $(g)$.

The lateral misalignments between the red and green planes is modelled as a function of the green plane, following equation 2 as:

$$
\left(\begin{array}{l}
\mathcal{C}^{r}\left(\mathbf{p}_{g}, \mathbf{c}_{r}\right)_{x} \\
\mathcal{C}^{r}\left(\mathbf{p}_{g}, \mathbf{c}_{r}\right)_{y}
\end{array}\right)=\left(\begin{array}{c}
c_{1} x_{g}+c_{2} x_{g} r_{g}^{2}+c_{3}\left(3 x_{g}^{2}+y_{g}^{2}\right)+2 c_{4} x_{g} y_{g} \\
c_{1} y_{g}+c_{2} y_{g} r_{g}^{2}+2 c_{3} x_{g} y_{g}+c_{4}\left(3 y_{g}^{2}+x_{g}^{2}\right)
\end{array}\right),
$$

and similarly for the difference between the blue and green planes. For each detected intersection point, two equations are formed. It is sufficient to follow these equations with respect to the red/green planes only:

$$
e\left(\hat{\theta}_{r}\right)=\left(\begin{array}{c}
e_{x}\left(\hat{\theta}_{r}\right) \\
e_{y}\left(\hat{\theta}_{r}\right)
\end{array}\right)=\left(\begin{array}{c}
\bar{u}_{g}+\mathcal{C}^{r}\left(\mathbf{p}_{g}, \mathbf{c}_{r}\right)_{x}-\bar{u}_{r} \\
\bar{v}_{g}+\mathcal{C}^{r}\left(\mathbf{p}_{g}, \mathbf{c}_{r}\right)_{y}-\bar{v}_{r}
\end{array}\right)
$$

where the parameter vector to be estimated is $\hat{\theta}_{r}=\left(\dot{x}, \dot{y}, a, c_{1}, c_{2}, c_{3}, c_{4}\right)$.

This function is minimised using a quadratic cost function $j(\theta)=e^{T}(\theta) Q e(\theta)$, where $Q$ is the estimated covariance, (assumed an identity matrix in this case). 
Performing a first order expansion of the error $e$ around the last iterative estimate $\hat{\theta}^{k}$, results in a Gauss-Newton scheme that can be iterated utilising many robust least square techniques Golub and Loan (1996):

$$
\hat{\theta}^{k+1}=\hat{\theta}^{k}-\lambda\left(\frac{\partial e^{T}\left(\hat{\theta}^{k}\right)}{\partial \theta} \frac{\partial e\left(\hat{\theta}^{k}\right)}{\partial \theta^{T}}\right)^{-1} \frac{\partial e^{T}\left(\hat{\theta}^{k}\right)}{\partial \theta} e\left(\hat{\theta}^{k}\right)
$$

where $\lambda \leq 1$ ensures a decrease in cost at each step. The partial derivatives used in the closed-form calculation are given as:

$$
\frac{\partial e\left(\hat{\theta}^{k}\right)}{\partial \theta^{T}}=\left(\begin{array}{c}
\frac{\partial e_{x}\left(\hat{\theta}^{k}\right)}{\partial \theta^{T}} \\
\frac{\partial e_{y}\left(\hat{\theta}^{k}\right)}{\partial \theta^{T}}
\end{array}\right)=\left(\begin{array}{c}
\frac{\partial e_{x}\left(\hat{\theta}^{k}\right)}{\partial \dot{x}}, \frac{\partial e_{x}\left(\hat{\theta}^{k}\right)}{\partial \dot{y}}, \frac{\partial e_{x}\left(\hat{\theta}^{k}\right)}{\partial a}, x_{g}, x_{g} r_{g}^{2}, 3 x_{g}^{2}+y_{g}^{2}, 2 x_{g} y_{g} \\
\frac{\partial e_{y}\left(\hat{\theta}^{k}\right)}{\partial \dot{x}}, \frac{\partial e_{y}\left(\hat{\theta}^{k}\right)}{\partial \dot{y}}, \frac{\partial e_{y}\left(\hat{\theta}^{k}\right)}{\partial a}, y_{g}, y_{g} r_{g}^{2}, 2 x_{g} y_{g}, 3 y_{g}^{2}+x_{g}^{2}
\end{array}\right)
$$

with

$$
\begin{aligned}
\left(\begin{array}{c}
\frac{\partial e_{x}\left(\hat{\theta}^{k}\right)}{\partial \dot{x}} \\
\frac{\partial e_{y}\left(\hat{\theta}^{k}\right)}{\partial \dot{x}}
\end{array}\right) & =\left(\begin{array}{c}
c_{1}+c_{2}\left(3 x_{g}^{2}+y_{g}^{2}\right)+6 c_{3} x_{g}+2 c_{4} y_{g} \\
2 c_{2} x_{g} y_{g}+2 c_{3} y_{g}+2 c_{4} x_{g}
\end{array}\right), \\
\left(\begin{array}{c}
\frac{\partial e_{x}\left(\hat{\theta}^{k}\right)}{\partial \dot{y}} \\
\frac{\partial e_{y}\left(\hat{\theta}^{k}\right)}{\partial \dot{y}}
\end{array}\right) & =\left(\begin{array}{c}
2 c_{2} x_{g} y_{g}+2 c_{3} y_{g}+2 c_{4} x_{g} \\
c_{1}+c_{2}\left(x_{g}^{2}+3 y_{g}^{2}\right)+2 c_{3} x_{g}+6 c_{4} y_{g}
\end{array}\right), \\
\left(\begin{array}{c}
\frac{\partial e_{x}\left(\hat{\theta}^{k}\right)}{\partial a} \\
\frac{\partial e_{y}\left(\hat{\theta}^{k}\right)}{\partial a}
\end{array}\right) & =\left(\begin{array}{c}
c_{1} u_{g}+3 c_{2} x_{g}^{2} u_{g}+6 c_{3} x_{g} u_{g}+2 c_{4} u_{g} y_{g} \\
2 c_{3} y_{g} u_{g}+2 c_{4} x_{g} u_{g}
\end{array}\right) .
\end{aligned}
$$

Equation 5 is iterated until $\hat{\theta}^{k+1}-\hat{\theta}^{k}$ falls below a preset threshold. The parameter vector can be simply initialised as $\theta_{r}^{0}=(-w / 2 s,-h / 2 s, 1,0,0,0,0)$, while the estimation algorithm generally converges within ten iterations. The estimated parameter uncertainties can be estimated using the Fisher information matrix:

$$
\mathbf{F}(\hat{\theta})=\frac{1}{\hat{\sigma}^{2}} \sum_{i=1}^{n} \frac{\partial e^{T}\left(\hat{\theta}^{k}\right)}{\partial \hat{\theta}} \frac{\partial e\left(\hat{\theta}^{k}\right)}{\partial \hat{\theta}^{T}}
$$

where $\hat{\sigma}^{2}=\frac{1}{n-m} \sum_{i=1}^{n} e\left(\hat{\theta}^{k}\right)^{2} . \mathbf{F}^{-1}(\hat{\theta})$ is then used to characterise the uncertainty in the parameters, by forming an estimate of the associated Standard Deviation (SD) as the square root of the $i^{\text {th }}$ diagonal element as:

$$
\mathrm{SD}\left(\hat{\Phi}_{i}\right)=\sqrt{\operatorname{diag}_{i}\left(\mathbf{F}^{-1}(\hat{\theta})\right.}
$$

\section{Experiments}

This section describes the experiments and results obtained relating to the measurement of LCA and its colour channel correlations in digital cameras. The integrity or stability of the estimated model parameters are also studied. 
Table 1

Description of the cameras used for the experiments

\begin{tabular}{|c|c|c|c|c|}
\hline & Make and Model & Focal & Resolution & CFA \\
\hline Cam 1 & Nikon E4500 & $7.8 \mathrm{~mm}$ & $2272 \times 1704$ & CYGM \\
Cam 2 & Fuji FinePix 6900 & $7.8 \mathrm{~mm}$ & $2832 \times 2128$ & RGB \\
Cam 3 & Fuji FinePix 6900 + WL-FX9 & $6.2 \mathrm{~mm}$ & $2832 \times 2128$ & RGB \\
\hline
\end{tabular}

Finally, the effects of LCA compensation in real images of controlled (calibration charts) and natural scenes are quantified and illustrated. Three different digital cameras are used in the experiments. Their properties are outlined in table 1.

\subsection{Measurement precision}

As outlined in Section 3.1, chessboard patterns are used to measure the content of LCA in images. The pattern used for calibration is shown in figure 2. No canonical coordinates are required for calibration, hence no precise constraints are needed on the planarity or precision of the pattern. A second lower density chessboard pattern (test image), shown in figure 2, is used for independent validation of the results.

The typical sub-pixel detection accuracy of the techniques outlined in section 3.1 are illustrated in figure 3 for the three cameras used in the experiments. Following extraction of the intersection locations on each colour plane of a black and white calibration chart the misalignments are calculated. These misalignments, representing the amount of LCA in the images, are presented in table 2 for the two types of chessboard patterns considered.
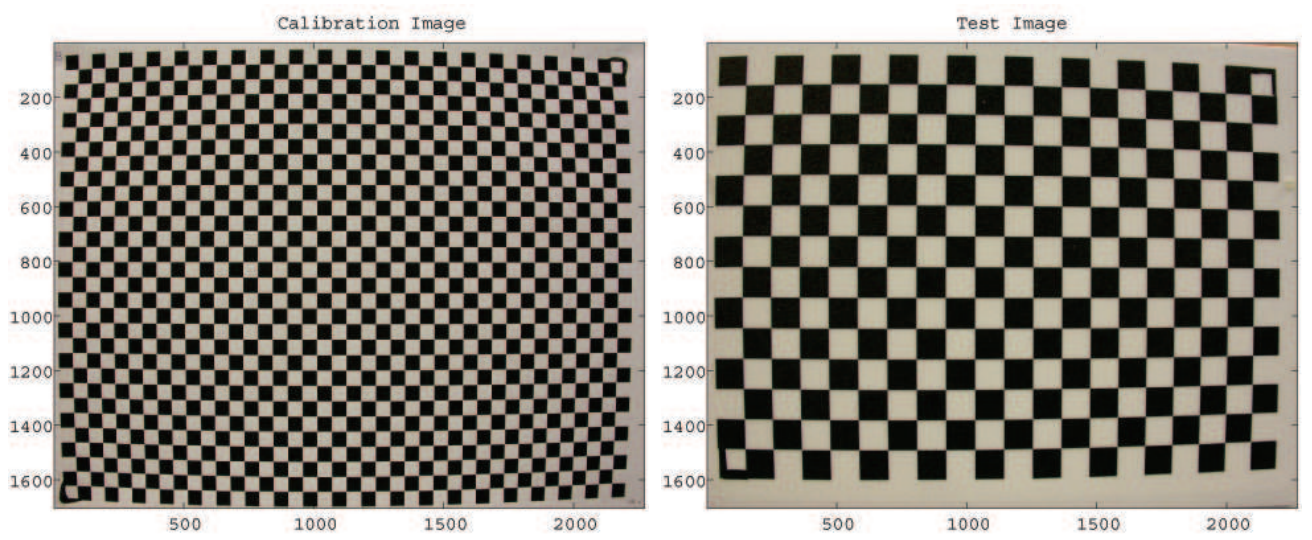

Fig. 2. Chessboard patterns used for calibration (calib image) and testing (test image) taken with cam 1 , see tables 2 and 4 . 

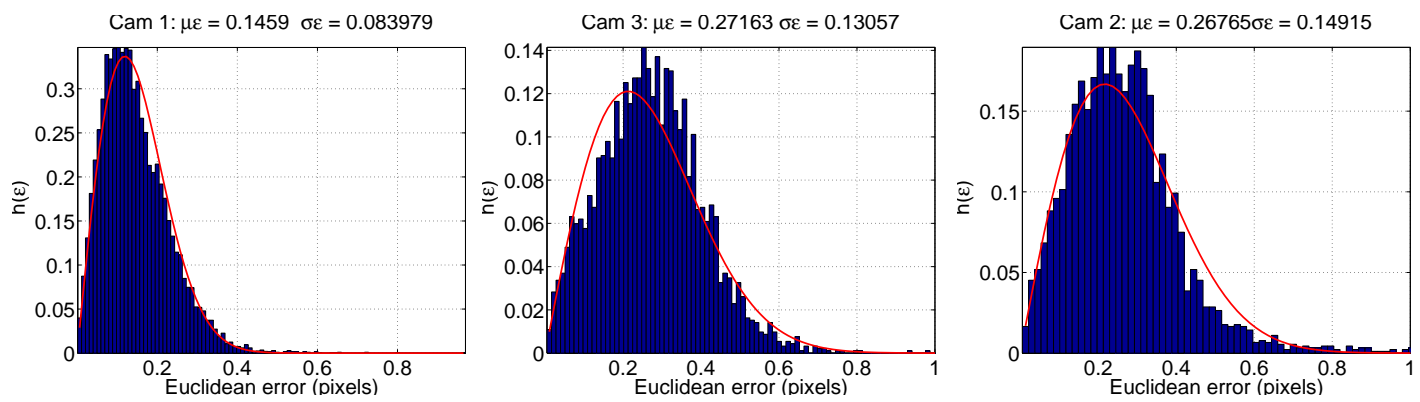

Fig. 3. Histogram of sub-pixel detection errors for three different cameras with their fitting with Rayleigh PDF. Errors are estimated using multiple shots of the calibration pattern.

Table 2

Colour plane misalignments (in pixels) before calibration in mean (standard deviation) format for three different cameras. $\mathrm{R} / \mathrm{G}$ and $\mathrm{B} / \mathrm{G}$ are the red and blue misalignments with reference to the green channel.

\begin{tabular}{|c|c|c|c|c|}
\hline & & Cam 1 & Cam 2 & Cam 3 \\
\hline \hline Calib & $\mathrm{R} / \mathrm{G}$ & $0.5707(0.2113)$ & $0.5496(0.2308)$ & $1.1834(0.4125)$ \\
\cline { 2 - 5 } Image & $\mathrm{B} / \mathrm{G}$ & $0.4110(0.2635)$ & $0.7374(0.6361)$ & $0.5665(0.3848)$ \\
\hline \hline \multirow{2}{*}{$\begin{array}{c}\text { Test } \\
\text { Image }\end{array}$} & $\mathrm{R} / \mathrm{G}$ & $0.5355(0.2225)$ & $0.5413(0.2035)$ & $0.9729(0.2866)$ \\
\cline { 2 - 5 } & $\mathrm{B} / \mathrm{G}$ & $0.4877(0.2925)$ & $1.1630(0.8971)$ & $0.8378(0.7956)$ \\
\hline
\end{tabular}

\subsection{Colour plane correlations}

As outlined in section 3, the interpolation process of the CFA in digital cameras may introduce additional correlations between colour channels. This has the potential to impact on the compensation for LCA by introducing additional colour dependent shifting. For example, assume there is a high correlation between the green channel and the blue channel due to the interpolation process. Thus, for a location in the blue channel, the observed LCA varies depending on the level of green. This would mean that the calibrated LCA model would only be applicable to colours similar to those that were used during calibration.

In order to investigate this idea, a set of experiments were designed. These consisted of comparing the location of a full spectrum (black and white) chessboard to that of a partially filtered one (coloured). The movement of the chessboard locations due to the removal of certain frequencies reveals the impact of the interpolation process. The experimental setup consists of a fixed camera and target with a white light source. This light is then filtered with colour filters to remove certain frequencies. The filters chosen are cyan, magenta and yellow, which block out, in turn, the red, green and blue frequencies. This configuration of filters is chosen as opposed to a RGB filtering scheme, as 
Table 3

Colour plane correlations in mean (SD) format. $G_{f}, R_{f}$ and $B_{f}$ are the measurement taken with certain colour removed by filtering.

\begin{tabular}{|l|c|c|c|c|}
\hline Filter & & Cam 1 & Cam 2 & Cam 3 \\
\hline \hline \multirow{2}{*}{ Cyan } & $G / G_{f}$ & $0.0883(0.0446)$ & $0.0984(0.0469)$ & $0.0949(0.0459)$ \\
& $B / B_{f}$ & $0.1443(0.0726)$ & $0.1982(0.1141)$ & $0.2406(0.1437)$ \\
\hline \multirow{2}{*}{ Magenta } & $R / R_{f}$ & $0.0966(0.0516)$ & $0.2142(0.0767)$ & $0.2366(0.0728)$ \\
& $B / B_{f}$ & $0.2256(0.1246)$ & $0.2949(0.1646)$ & $0.3588(0.2020)$ \\
\hline \multirow{2}{*}{ Yellow } & $R / R_{f}$ & $0.1490(0.0534)$ & $0.1386(0.0618)$ & $0.1302(0.0662)$ \\
& $G / G_{f}$ & $0.0550(0.0323)$ & $0.1506(0.0482)$ & $0.0887(0.0428)$ \\
\hline
\end{tabular}

we consider that LCA only visibly manifests itself with reference to another colour, and is thus most appropriate.

The results of these experiments are presented in table 3. These show that the correlations due to the lack of blue and red are around the level of the detection accuracy. The vector plots of these differences reveal no discernable chromatic like dependence. The differences due to the absence of green are slightly larger, while their vector plots reveal a small (in comparison to the measured LCA in table 2) chromatic like dependence. We conclude that the interpolation does induce an additional chromatic error, but to a negligible degree with regard to the available measurement accuracy and the LCA content. Therefore, considering the relative magnitudes, it can be expected that the LCA calibrated using a black and white pattern will be applicable to any colour. This is borne out in the experiments with natural scenes in section 4.3. The varying correlations of different interpolation methods is beyond the scope of this paper.

\subsection{Chromatic compensation}

Following calibration using a black and white calibration chart, the known LCA models are used to warp the colour planes so as to register the red and blue colour planes with the green channel. The Euclidean registration residuals remaining following this re-registration are presented in table 4 , showing a significant decrease in misalignments. These residuals are of a similar magnitude to the sub-pixel detection accuracy, thus validating both the proposed LCA model and the effectiveness of the proposed calibration algorithm.

As described in section 2, some lenses exhibit a significant tangential LCA component. The results presented in table 5 show the Euclidean registration residuals following compensation based on a model without tangential ele- 
Table 4

Colour plane misalignments (in pixels) following calibration and colour plane warping in mean (standard deviation) format for three different cameras.

\begin{tabular}{|c|c|c|c|c|}
\hline & & Cam 1 & Cam 2 & Cam 3 \\
\hline \hline \multirow{2}{*}{$\begin{array}{c}\text { Calib } \\
\text { Image }\end{array}$} & $\mathrm{R} / \mathrm{G}$ & $0.1202(0.0636)$ & $0.1401(0.0733)$ & $0.1846(0.0722)$ \\
\cline { 2 - 5 } & $\mathrm{B} / \mathrm{G}$ & $0.1376(0.0734)$ & $0.1658(0.0947)$ & $0.1543(0.0925)$ \\
\hline \hline \multirow{2}{*}{$\begin{array}{c}\text { Test } \\
\text { Image }\end{array}$} & $\mathrm{R} / \mathrm{G}$ & $0.1788(0.1062)$ & $0.1625(0.0784)$ & $0.2044(0.1149)$ \\
\cline { 2 - 5 } & $\mathrm{B} / \mathrm{G}$ & $0.1879(0.1110)$ & $0.3092(0.2146)$ & $0.3202(0.2419)$ \\
\hline
\end{tabular}

Table 5

Colour plane misalignments (in pixels) following calibration and warping using a model without tangential elements in mean (standard deviation) format for three different cameras.

\begin{tabular}{|c|c|c|c|c|}
\hline & & Cam 1 & Cam 2 & Cam 3 \\
\hline \hline \multirow{2}{*}{$\begin{array}{c}\text { Calib } \\
\text { Image }\end{array}$} & $\mathrm{R} / \mathrm{G}$ & $0.1828(0.0904)$ & $0.1615(0.0858)$ & $0.2196(0.1208)$ \\
\cline { 2 - 5 } & $\mathrm{B} / \mathrm{G}$ & $0.2131(0.1117)$ & $0.1805(0.1087)$ & $0.1507(0.0754)$ \\
\hline \hline \multirow{2}{*}{$\begin{array}{c}\text { Test } \\
\text { Image }\end{array}$} & $\mathrm{R} / \mathrm{G}$ & $0.1864(0.1110)$ & $0.2022(0.1019)$ & $0.1886(0.1449)$ \\
\cline { 2 - 5 } & $\mathrm{B} / \mathrm{G}$ & $0.2071(0.1334)$ & $0.3670(0.3070)$ & $0.3761(0.3029)$ \\
\hline
\end{tabular}

ments. The increase in these residuals compared with those of the full calibration model indicate that although radial chromatic aberration is predominant, there is a varying element of tangential aberration based on the lens employed. The inclusion of tangential elements in the LCA description gives a more general and accurate model of lateral chromatic aberration in an image.

Full details of the colour plane misalignments before and after calibration are presented for one example (Cam 1) from table 2 and 4 . Figure 4 shows the distribution of colour plane misalignments before and after compensation for LCA for the calibration pattern in figure 2. The corresponding Euclidean vector representation of these misalignments for the test image, before and after compensation, are illustrated in figure 5. These show that the remaining misalignments are random in nature (with magnitude similar to the detection noise), indicating the successful modelling and compensation of LCA.

To assess the improvement in image quality a selection of examples are shown for each camera referred to in tables 2 and 4 . For each camera a region of interest (ROI) is selected in the test image and an outdoor scene image. Images taken with Cam 1 are presented in figures 6 and 7, Cam 2 in figures 8 and 9, while Cam 3 examples are presented in figures 10 and 11. The associated colour histograms for the test ROI's of figures 6,8 and 10 show that for the uncorrected image, two colour paths exist between the black and white squares of the test pattern. This is due to the additional colour fringing introduced 
by the LCA around regions of high contrast. The colour histograms for the corrected images show that following compensation there is only one colour path between dark and bright squares, indicating the successful removal of LCA from these images. The real examples of figures 7, 9 and 11 show a similar behaviour. Additional colour paths can be seen in the original images, while following calibration and compensation these extra colors are removed. These results clearly indicate that the proposed method of automatically calibrating and removing LCA in images leads to a significant increase in image quality.

\subsection{Model parameter analysis}

To determine the suitability of the model, in terms of redundant parameters, and its stability, it is useful to look at the parameter uncertainties. These measures may be computed directly from the iterative estimation scheme as described in equation 7 . In order to concisely describe these results two extreme examples are taken from the calibration data in section 4.3. These are the red/green alignments in Cam 1 and Cam 3. The parameter values and uncertainties are presented in table 6 . The parameter $c_{1}$, related to the lateral colour distortion, takes a large role in the calibration in Cam 1. However, in all estimations its estimated uncertainty value remains low indicating a stable system of equations. Parameter $c_{2}$, related to the chromatic variation of distortion proves useful for all calibrations and also exhibits low uncertainties. The decentering parameters $c_{3}$ and $c_{4}$ play a variable role, clearly related to the camera or lens employed. The centre point estimates and uncertainties are presented for completeness.

Table 6

Parameter values and uncertainties for the selection of reg/green calibration with Cam 1 and Cam 3

\begin{tabular}{|c|c|c|}
\hline Parameter & Cam 1 & Cam 3 \\
\hline$c_{1} \times 10^{-3}$ & $82.730(0.0094)$ & $2.021(0.0152)$ \\
$c_{2} \times 10^{-3}$ & $-11.164(0.0142)$ & $-4.970(0.1023)$ \\
$c_{3} \times 10^{-4}$ & $-0.0693(0.0252)$ & $2.188(0.0633)$ \\
$c_{4} \times 10^{-4}$ & $-0.0716(0.0349)$ & $1.111(0.0891)$ \\
\hline$u_{o}$ & $1163(5.33)$ & $1335(4.13)$ \\
$v_{o}$ & $903(5.38)$ & $1074(4.18)$ \\
\hline
\end{tabular}



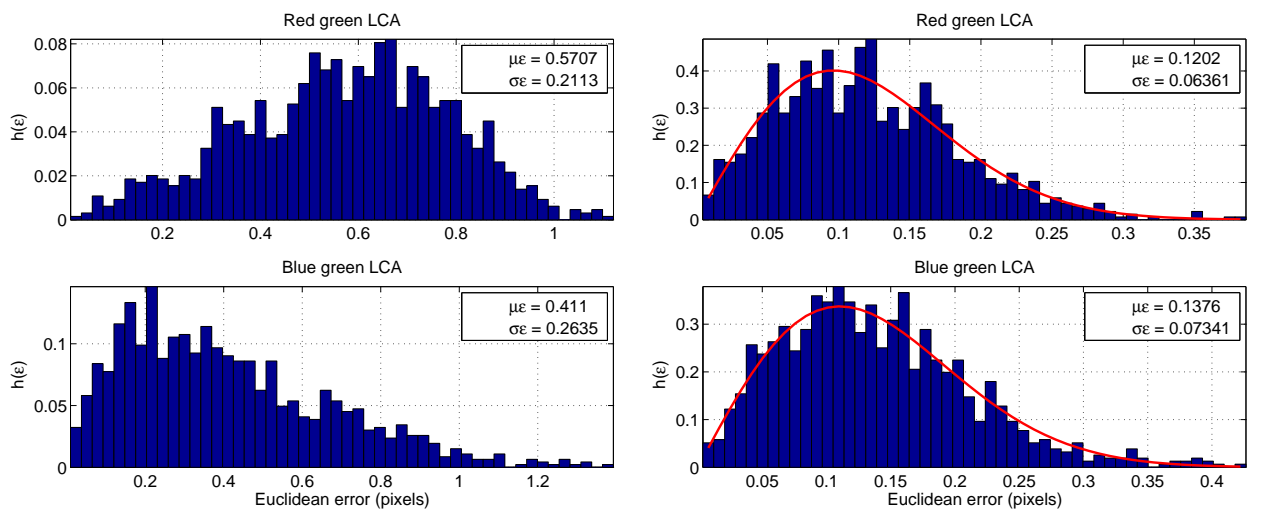

Fig. 4. Histograms of Euclidean misalignments computed for chessboard intersections on the calibration image with Cam 1. Left column shows the R/G and B/G differences before compensation, while the right column shows those detected following calibration with fitted Rayleigh PDF's.
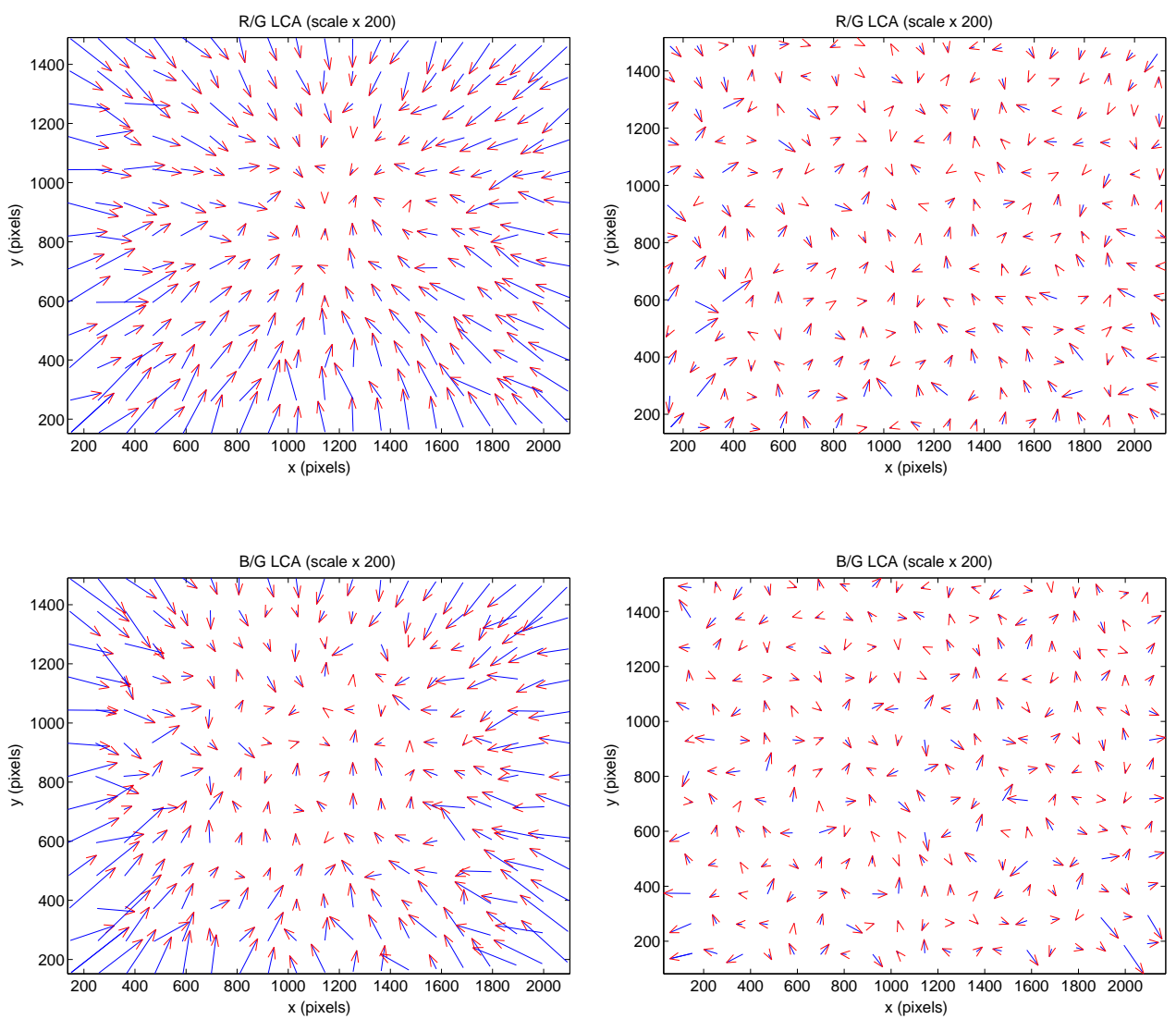

Fig. 5. Euclidean vector plots of colour plane misalignments before (left column) and after (right column) LCA compensation, evaluated on the test chessboard pattern with Cam 1. 


\section{Conclusion}

This paper proposes a new model based method of compensating for lateral chromatic aberration in images, offering a usable alternative to active lens control techniques and data interpolation methods. The main contributions of this work are the derivation of lateral chromatic aberration models and their subsequent parameter estimation techniques. These calibration techniques are easy to use, based on a single view of a chessboard pattern without any strict planarity constraints. This fully automated method is presented with full partial derivatives allowing faster and simpler estimation. Compensated images are formed by re-sampling the originals based on these calibrated models generating higher quality aberration free images. Model validation is carried out indicating strong global agreement with detected LCA. The effects of colour plane correlations are measured and shown to be of negligible magnitude with respect to the measurement accuracy and the LCA content. Examples on selections of real images demonstrate the higher quality achievable with such aberration removal. These show that the additional colours that LCA introduces are removed following compensation with the proposed methods, ultimately giving superior quality colour images. 

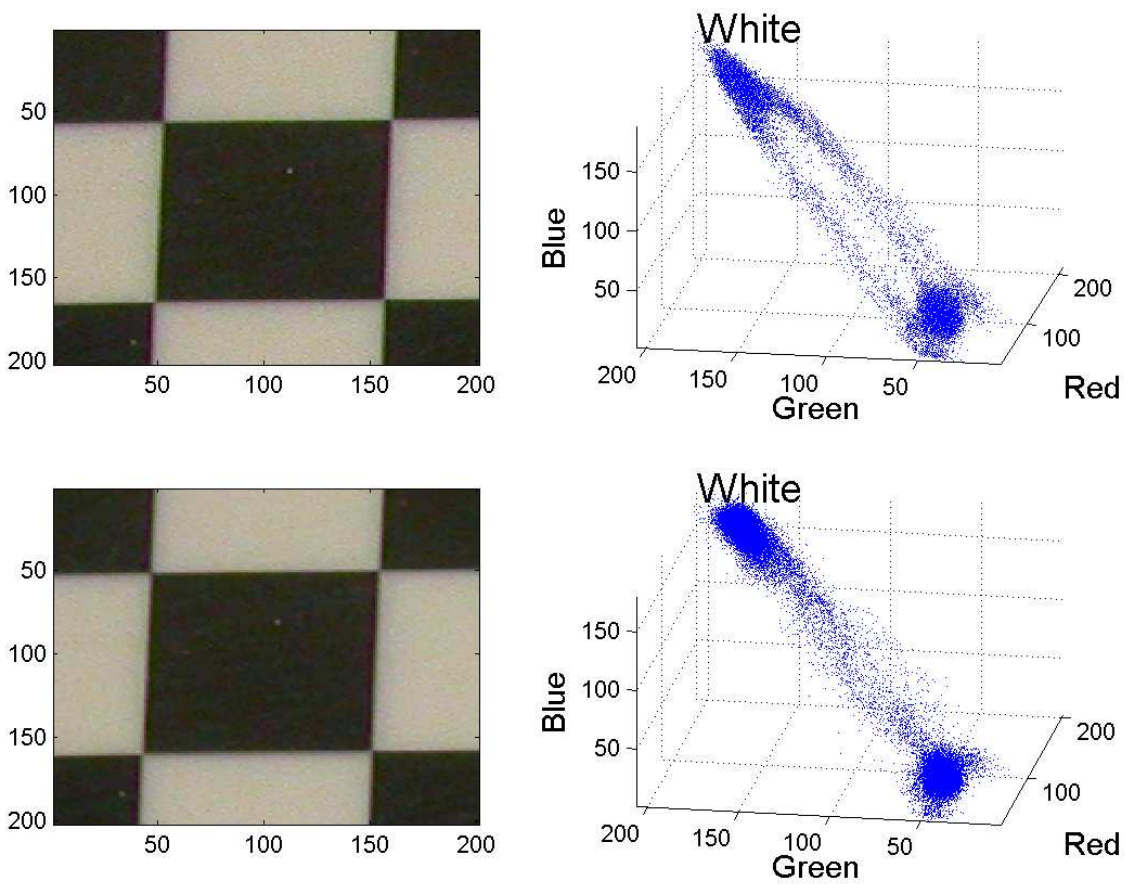

Fig. 6. Test image for Cam 1. Top row shows crop with associated colour histogram before compensation. Two additional colour paths are formed by the colour fringing between dark and bright regions. Second row shows the corresponding LCA compensated crop, where the colour fringing has been cancelled.
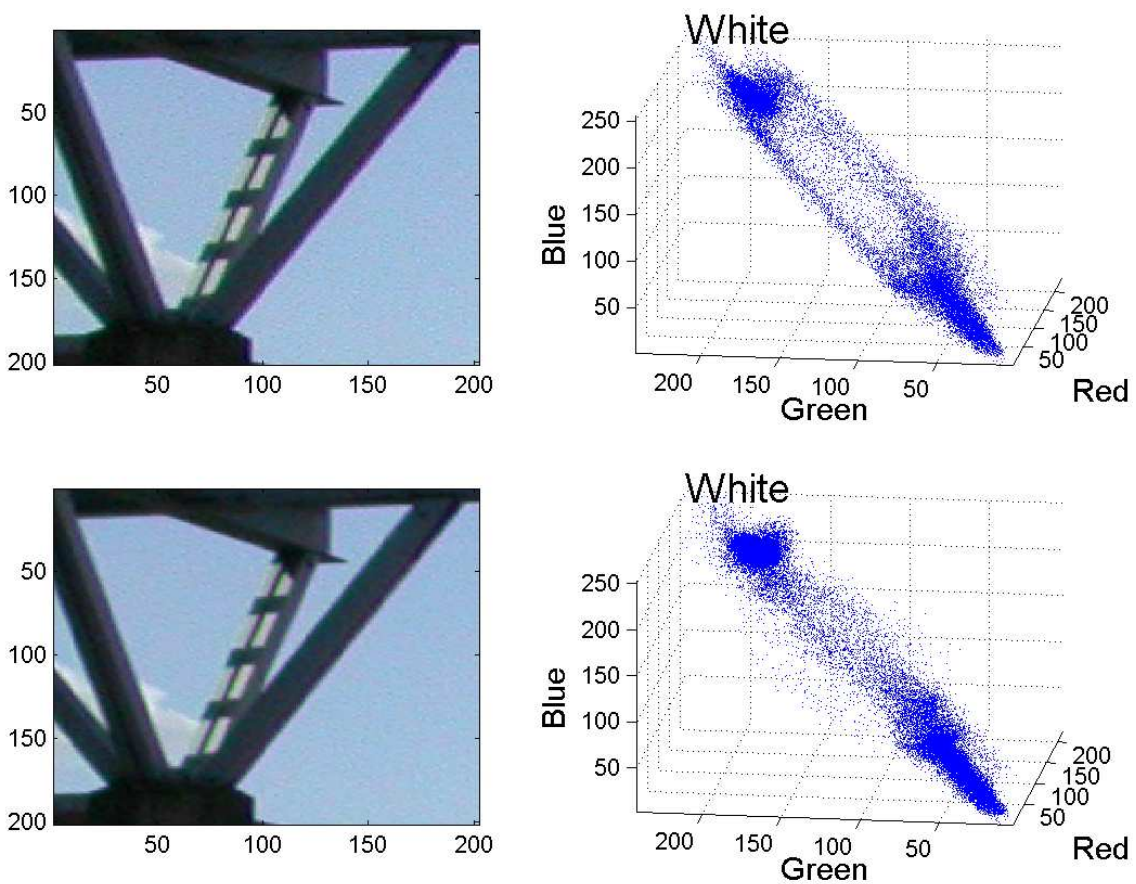

Fig. 7. Outdoor image for Cam 1. Top row shows crop with associated colour histogram before compensation. Additional colour paths are formed by the colour fringing. Second row shows the corresponding LCA compensated crop, where the colour fringing has been removed. 

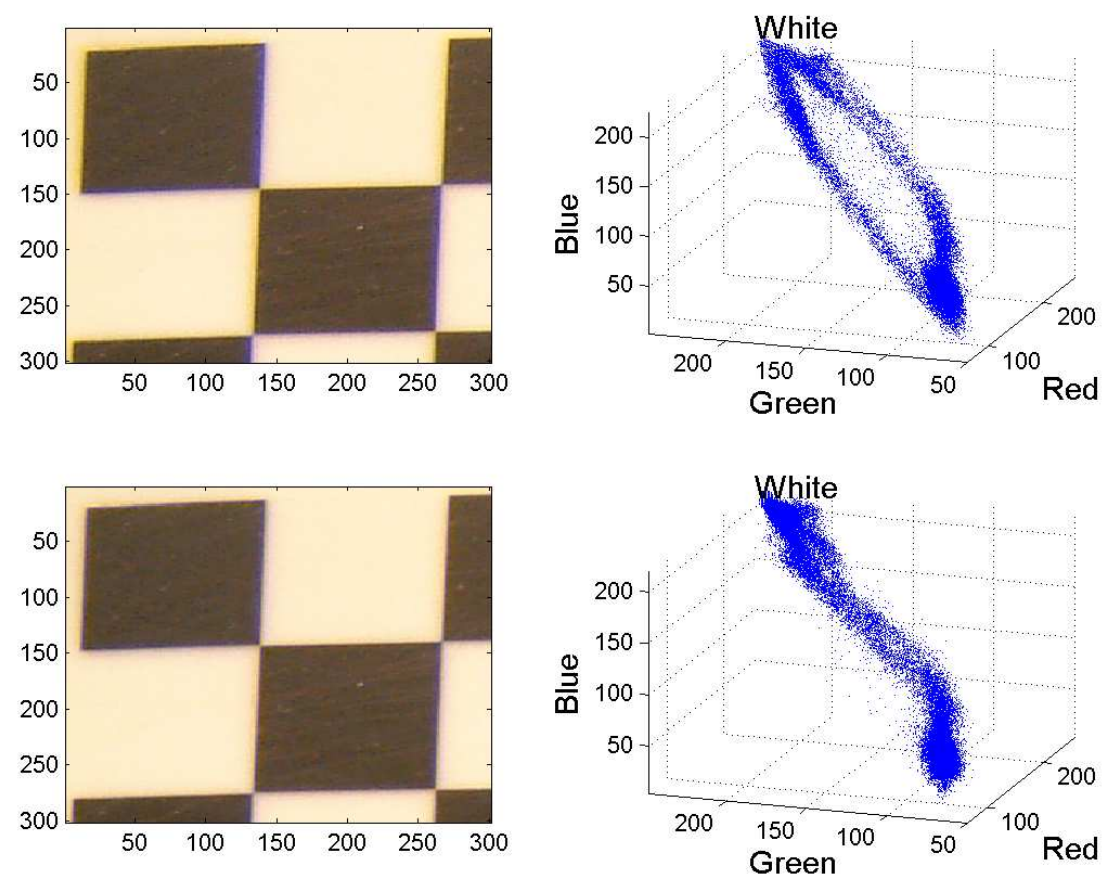

Fig. 8. Test image for Cam 2. Top row shows crop with associated colour histogram before compensation. Two additional colour paths are formed by the colour fringing between dark and bright regions. Second row shows the corresponding LCA compensated crop, where the colour fringing has been cancelled.
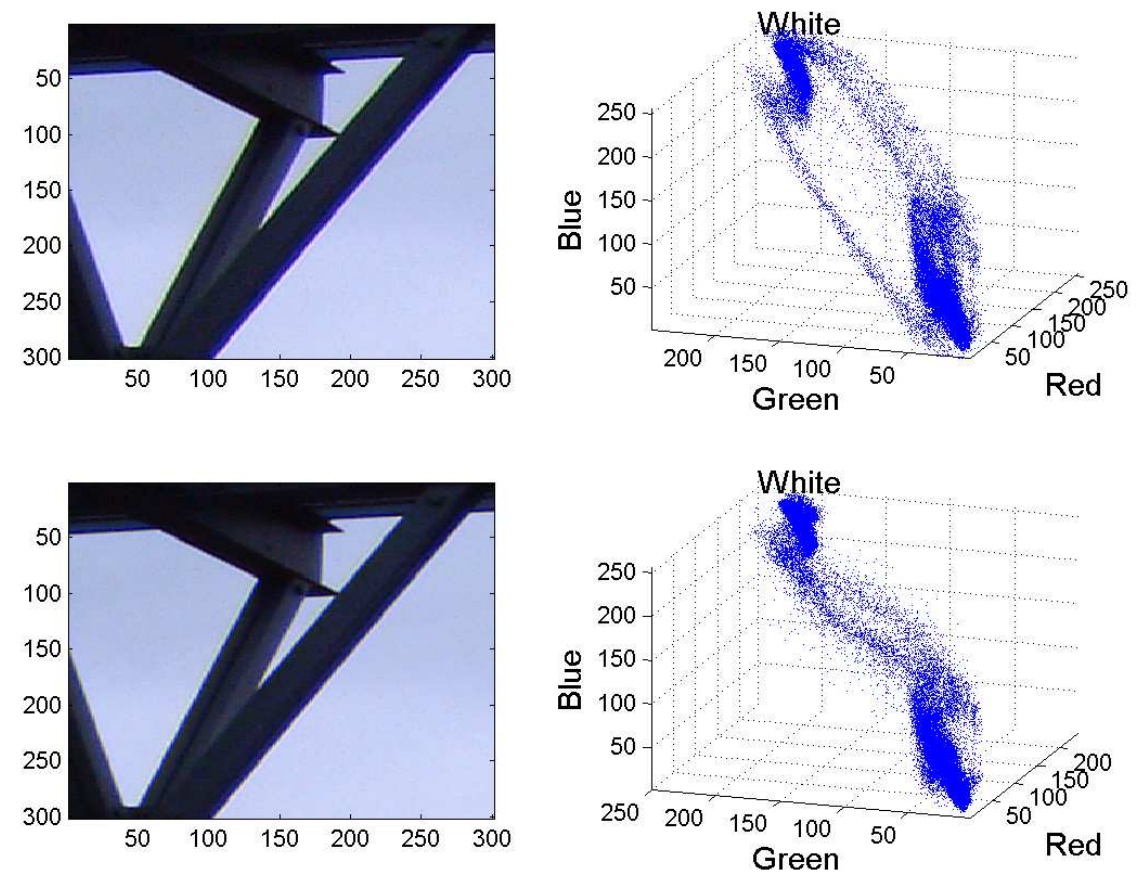

Fig. 9. Outdoor image for Cam 2. Top row shows crop with associated colour histogram before compensation. Additional colour paths are formed by the colour fringing. Second row shows the corresponding LCA compensated crop, where the colour fringing has been removed. 

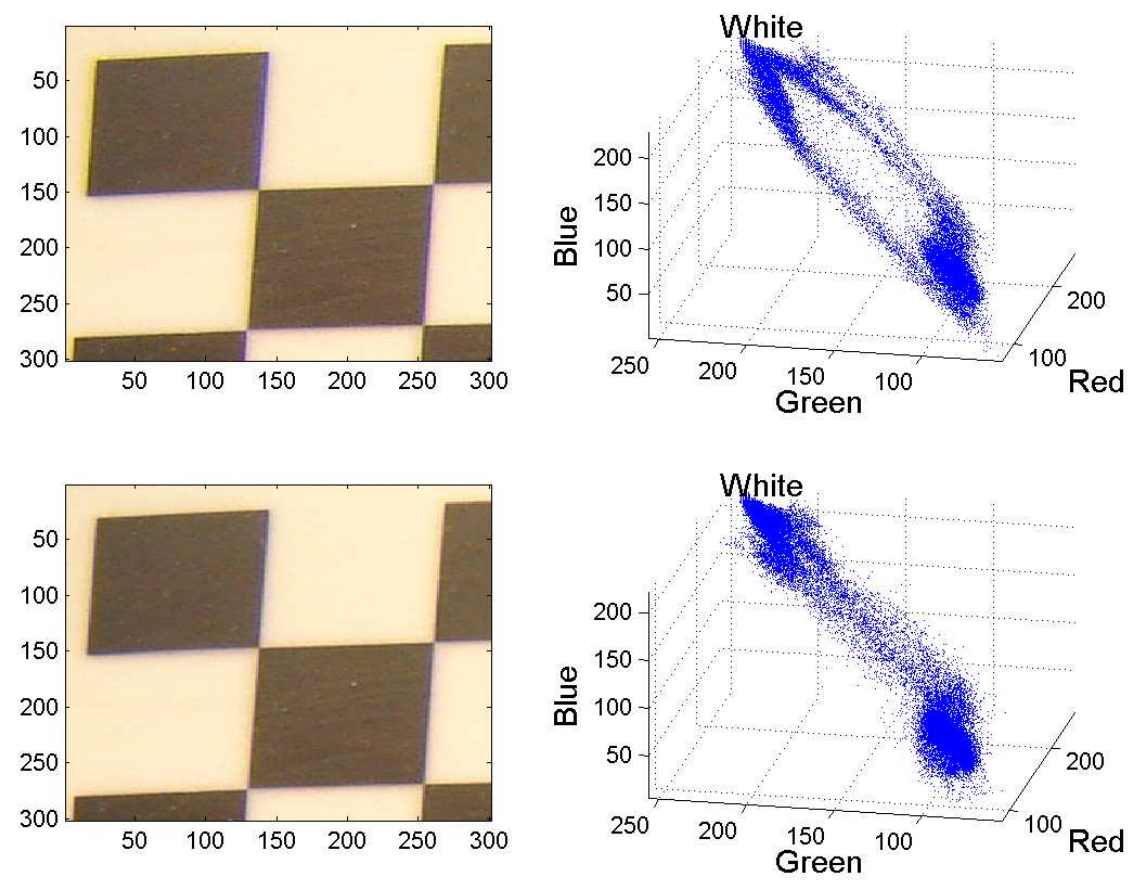

Fig. 10. Test image for Cam 3. Top row shows crop with associated colour histogram before compensation. Two additional colour paths are formed by the colour fringing between dark and bright regions. Second row shows the corresponding LCA compensated crop, where the colour fringing has been cancelled.
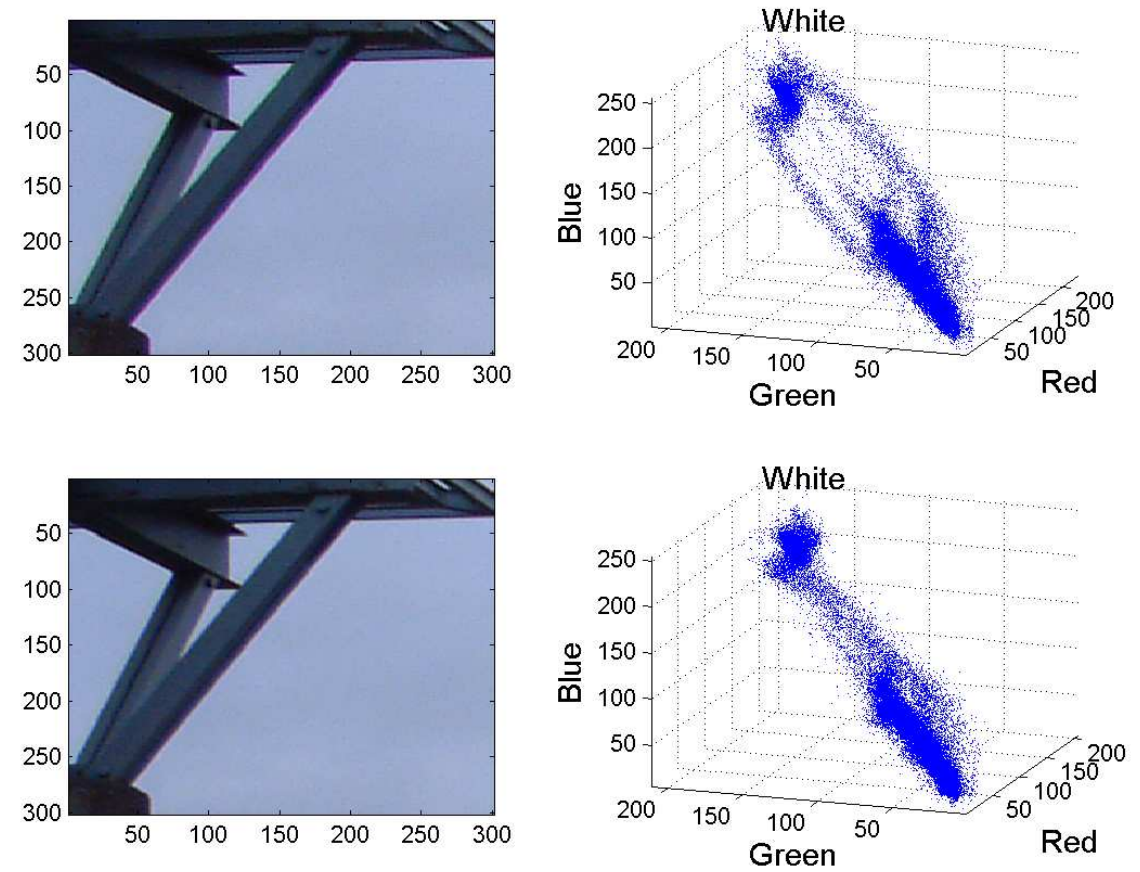

Fig. 11. Outdoor image for Cam 3. Top row shows crop with associated colour histogram before compensation. Additional colour paths are formed by the colour fringing. Second row shows the corresponding LCA compensated crop, where the colour fringing has been removed. 


\section{References}

Born, M., Wolf, E., 1980. Principles of Optics, sixth Edition. Permagon.

Boult, T.E.; Wolberg, G., 1992. Correcting chromatic aberrations using image warping. In: IEEE Conference on Computer Vision and Pattern Recognition. pp. $684-687$.

Brown, D. C., 1966. Decentering distortion of lenses. Photogrammetric engineering 32 (3), 444-462.

Conrady, A., 1919. Decentered lens systems. Monthly notices of the royal astronomical society 79, 384-190.

Driscoll, W. G., 1978. Handbook of optics. McGraw Hill.

Freitag, B., Kujawa, S., Mul, P., Ringnalda, J., Tiemeijer, P., 2005. Breaking the spherical and chromatic aberration barrier in transmission electron microscopy. Ultramicroscopy 102 (3), 209-214.

Garcia, J., Sanchez, J. M., Orriols, X., Binefa, X., 2000. Chromatic aberration and depth extraction. In: International Conference on Pattern Recognition. Vol. 1. pp. $762-765$.

Golub, G. H., Loan, C. F. V., 1996. Matrix Computation, 3rd Edition. Johns Hopkins University Press.

Jackowski, M., Goshtasby, A., Bines, S., Roseman, D., Yu, C., 1997. Correcting the geometry and color of digital images. IEEE Transactions on Pattern Analysis and Machine Intelligence 19 (10), 1152-1158.

Jain, R., Kasturi, R., Schunck, B. G., 1995. Machine vision. McGraw-Hill.

Kingslake, R., 1978. Lens Design fundamentals. academic press.

Kuzubek, M., Matula, P., 2000. An efficient algorithm for measurement and correction of chromatic aberrations in fluorescence microscopy. Journal of Microscopy 200 (3), 206-217.

Lucchese, L., Mitra, S. K., 2002. Using saddle points for subpixel feature detection in camera calibration targets. In: Asia-Pacific Conference on Circuits and Systems. Vol. 2. pp. $191-195$.

Popescu, A. C., Farid, H., 2005. Exposing digital forgeries in color filter array interpolated images. IEEE Transactions on Signal Processing 53 (10), 39483959.

Ramanath, R., Snyder, W. E., Bilbro, G. L., 2002. Demisaicking methods for bayer color arrays. Journal of Electronic Imaging 11 (3), 306-315.

Willson, R., 1994. Modeling and calibration of automated zoom lenses. Ph.D. thesis, Carnegie Mellon University.

Willson, R. G., Shafer, S. A., 1991. Active lens control for high precision computer imaging. In: IEEE International conference on robotics and automation. pp. 2063-2070. 